\title{
Flexible polyimide-based hybrid opto- electric neural interface with 16 channels of micro-LEDs and electrodes
}

\author{
Bowen Ji ${ }^{1}$, Zhejun Guo ${ }^{1}$, Minghao Wang ${ }^{1}$, Bin Yang ${ }^{1}$, Xiaolin Wang ${ }^{1}$, Wen Li ${ }^{2}$ and Jingquan Liu ${ }^{1}$
}

\begin{abstract}
In this paper, a polyimide-based flexible device that integrates 16 micro-LEDs and $16 \mathrm{IrO}_{x}$-modified microelectrodes for synchronous photostimulation and neural signal recording is presented. The $4 \times 4$ micro-LEDs (dimensions of $220 \times$ $270 \times 50 \mathrm{\mu m}^{3}, 700 \mu \mathrm{m}$ pitch) are fixed in the SU-8 fence structure on a polyimide substrate and connected to the leads via a wire-bonding method. The recording electrodes share a similar fabrication process on the polyimide with 16 microelectrode sites $\left(200 \mu \mathrm{m}\right.$ in diameter and $700 \mu \mathrm{m}$ in pitch) modified by iridium oxide $\left(\mathrm{IrO}_{x}\right)$. These two subparts can be aligned with alignment holes and glued back-to-back by epoxy, which ensures that the light from the LEDs passes through the corresponding holes that are evenly distributed around the recording sites. The long-term electrical and optical stabilities of the device are verified using a soaking test for 3 months, and the thermal property is specifically studied with different duty cycles, voltages, and frequencies. Additionally, the electrochemical results prove the reliability of the $\mathrm{IrO}_{x}$-modified microelectrodes after repeated pressing or friction. To evaluate the tradeoff between flexibility and strength, two microelectrode arrays with thicknesses of 5 and $10 \mu \mathrm{m}$ are evaluated through simulation and experiment. The proposed device can be a useful mapping optogenetics tool for neuroscience studies in small (rats and mice) and large animal subjects and ultimately in nonhuman primates.
\end{abstract}

\section{Introduction}

Considerable progress has been made in the last decade in optogenetics to manipulate specific circuits by excitation or inhibition of a specific neuron type with the expression of light-sensitive ion channels or ion pumps. This method is critically important in neuroscience research because of its capabilities in sophisticated functional studies in neural systems ${ }^{1,2}$. To deliver light to the targeted brain area, the conventional method is to insert an optical fiber into the brain tissue with an attachment at the opposite end to a remotely located laser or lightemitting diode (LED) $)^{3,4}$. However, the light sources have low spatial resolution due to the limited number of beams

\footnotetext{
Correspondence: Jingquan Liu (jqliu@sjtu.edu.cn)

${ }^{1}$ National Key Laboratory of Science and Technology on Micro/Nano Fabrication, Department of Micro/Nano Electronics, Shanghai Jiao Tong University, Shanghai 200240, China

${ }^{2}$ Electrical and Computer Engineering Department, Michigan State University, East Lansing, MI 48824, USA
}

and the relatively imprecise positioning of the sources. To solve this problem, LED arrays ${ }^{5}$ or optical waveguide arrays $^{6,7}$ have been applied. Additionally, because optical fibers can restrict the movement of the animal or even lead to entanglement, the ultra-miniaturized wirelesspowered LED array is a superior choice ${ }^{8,9}$.

The subdural or epidural electrocorticography (ECoG) signals have been brought into focus in both research and application of functional and cognitive neuroscience due to the advantages of capturing broader band neural activity with higher spatial resolution ${ }^{10}$ and carrying more motor, cognitive, and language information for brain-computer interface control than electroencephalography ${ }^{11}$. Thus, ECoG is far more valuable and reliable with a high level of performance. To acquire ECoG signals, a high-density microelectrode array is usually utilized to record local field potentials from a large-area cortical surface at mesoscopic scales ${ }^{12-14}$. As the number of microelectrode sites increases, more

\section{(c) The Author(s) 2018}

(c) (i) Open Access This article is licensed under a Creative Commons Attribution 4.0 International License, which permits use, sharing, adaptation, distribution and reproduction cc) in any medium or format, as long as you give appropriate credit to the original author(s) and the source, provide a link to the Creative Commons license, and indicate if changes were made. The images or other third party material in this article are included in the article's Creative Commons license, unless indicated otherwise in a credit line to the material. If material is not included in the article's Creative Commons license and your intended use is not permitted by statutory regulation or exceeds the permitted use, you will need to obtain permission directly from the copyright holder. To view a copy of this license, visit http://creativecommons.org/licenses/by/4.0/. 

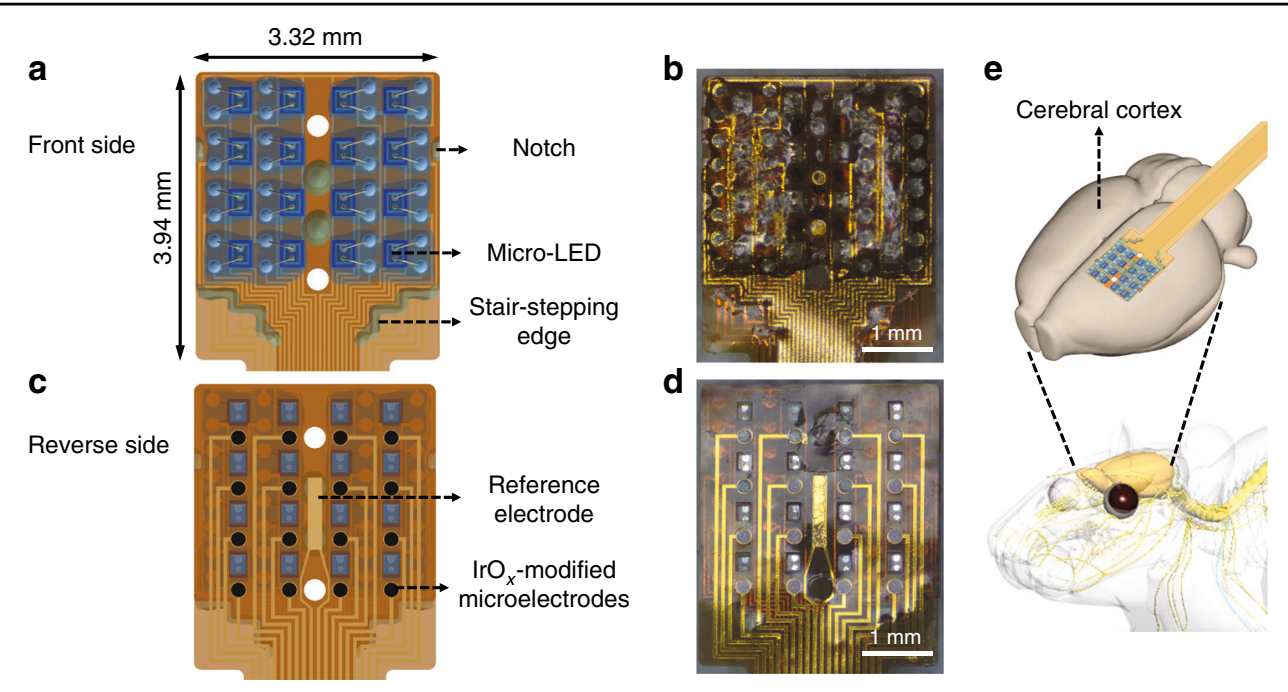

Fig. 1 Design schematic of the opto-electric neural interface. a Schematic diagram and $\mathbf{b}$ photograph of the front side of the assembled device with 16 micro-LEDs on the top, $\mathbf{c}$ schematic diagram and $\mathbf{d}$ photograph of the reverse side of the assembled device with $16 \mathrm{IrO}_{x}$-modified microelectrodes on the top, and $\mathbf{e}$ the sketch of the device attached on the cerebral cortex of a rat

information will be stored during multichannel recording. The entire size of the microelectrode array varies with the target cortical area of different animal species from mice ${ }^{15}$ and rats $^{16}$ to primates ${ }^{17}$. However, few researchers have combined the micro-LED array with the microelectrode array for synchronous photostimulation and ECoG signal recording of nervous tissues ${ }^{5,18}$ in neural circuit research.

Advances in microelectromechanical system (MEMS) technologies have enabled precise fabrication of multichannel micro-LED arrays on flexible substrates ${ }^{5,19}$. Microelectrode arrays can also be obtained using similar MEMS fabrication approaches $5,13,20$. Compared to our previous work $^{21}$, this paper has completely changed the device design by increasing the channel count from 3 micro-LEDs and 4 recording sites to 16 micro-LEDs and 16 recording sites. Now, this device is capable of simultaneous, high-resolution optical stimulation and electrical recording of a larger cortical area. In this work, we first describe the practical design for the application of the device on the cortical surface of a rat, as well as the detailed processes of the fabrication, including the respective flows of the microLED array and the microelectrode array. To ensure that the luminous surface of the micro-LEDs faces the same direction as the microelectrodes, wire-bonding technology is utilized for the electrical conduction instead of the lowmelting-point solder bonding ${ }^{5}$ or the flip-chip bonding ${ }^{22}$. A key step in the wire-bonding connection is the silver pasteaided reinforcement that significantly improves wirebonding strength and yield on the flexible polymer substrate. Accurate positioning of the micro-LEDs over the $4 \times$ 4 holes on the microelectrode array is assisted by two temporary needles that penetrate through the identical alignment holes on the two sub-arrays into the poly-dimethyl-siloxane (PDMS) substrate.

For long-term implantation, the reliability of the device should be taken into consideration. Accordingly, we conducted a soaking test in the phosphate buffer solution (PBS) for 3 months and found little degradation in the electrical or optical performance. For the recording microelectrodes, a comparison of the electrochemical properties was performed before and after 5000 iterations of pressing or friction, and the performance declined very little as well. Moreover, in view of the potential thermal damage to the local tissue due to the heating of the microLED, the temperature increase of the micro-LED was studied specifically with different duty cycles $(0.1-0.9)$, voltages $(2.5-3.9 \mathrm{~V})$, and frequencies $(1-50 \mathrm{~Hz})$, which can serve as a reference for safe optical stimulation. Finally, the flexibility and mechanical strength of two specimens with different thicknesses $(5$ and $10 \mu \mathrm{m})$ were evaluated by simulation and experiment, in which the thinner specimen $(5 \mu \mathrm{m})$ is suitable for acute animal experiments due to better flexibility, whereas the thicker specimen $(10 \mu \mathrm{m})$ is more durable and reliable in longterm implantation of free-moving rats.

\section{Materials and methods}

\section{Design of the opto-electric neural interface}

The design objectives of this neural interface are optoelectric integration, mapping of the targeted brain area of a rat, and reliability for long-term use. The design schematic is shown in Fig. 1. This neural interface is composed of two individual parts with compatible fabrication 

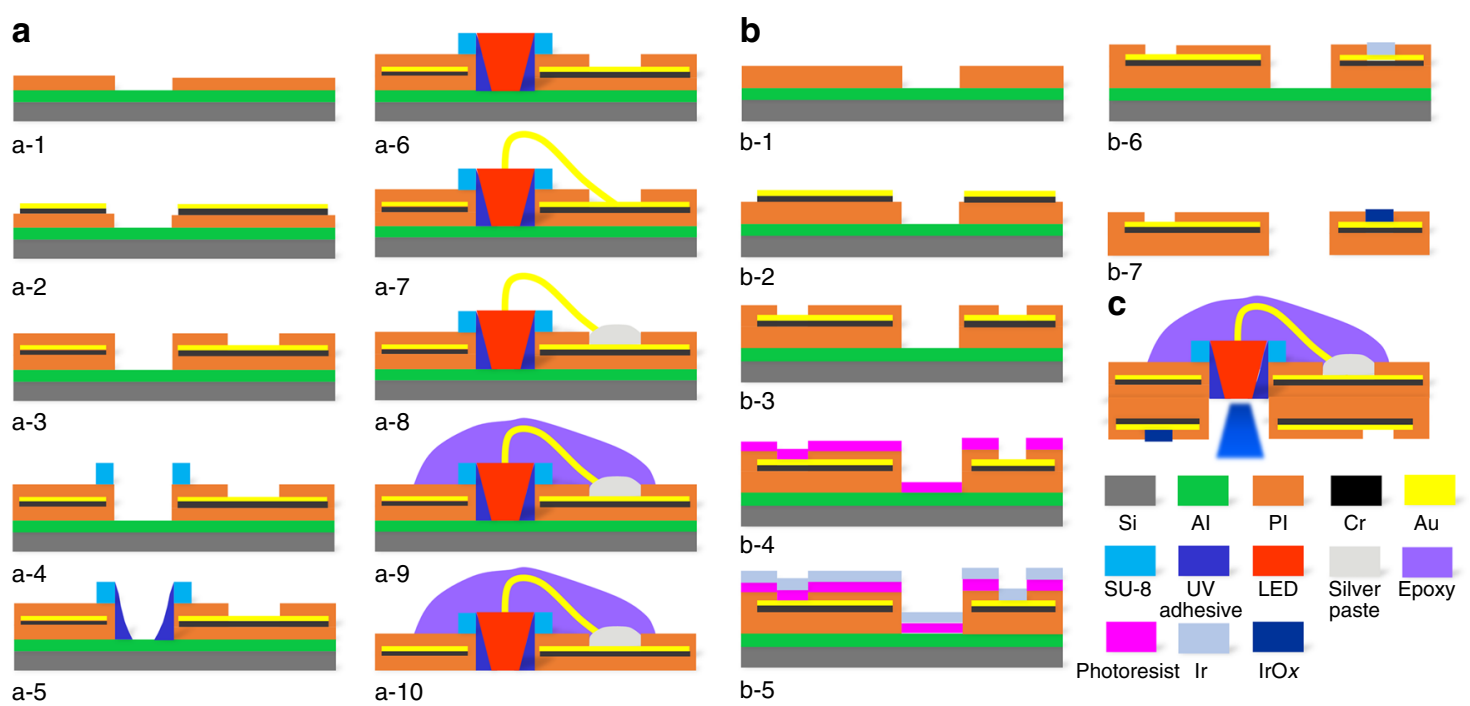

Fig. 2 Fabrication processes. a The micro-LED array and $\mathbf{b}$ the micro-ECoG electrode array based on polyimide substrate. $\mathbf{c}$ Assembled device with the micro-LED array stacked on the micro-ECoG electrode array

processes, the $4 \times 4$ micro-LED array for optical stimulation, and the $4 \times 4$ microelectrode array for neural recording.

As illustrated in Fig. 1a, the overall size of the assembled device is $3.32 \times 3.94 \mathrm{~mm}^{2}$, which matches the size of the cerebral hemisphere in rats. The photograph in Fig. 1b shows the front side of the fabricated device. In this assembly, the footprint of the micro-LED array is smaller than that of the microelectrode array with a stair-stepping end edge to apply the epoxy along this edge for adhesion of the two sub-arrays. Four circular holes are evenly distributed in the middle of the micro-LED array footprint, in which the two middle holes are used for dispensing the adhesive and the two side holes are used for the alignment with the same two holes on the microelectrode array. Additionally, two notches are symmetrically located at the edge of the footprint of the micro-LED array for dispensing the adhesive as well. To achieve relatively high resolution, according to the optimal spacing of the subdural, epidural, and scalp electrodes for rats as suggested by Slutzky and Kwon ${ }^{23,24}$, the pitch between the two neighboring micro-LEDs and for the two adjacent microelectrode sites is designed as $700 \mu \mathrm{m}$.

Sixteen $(4 \times 4)$ bare micro-LED chips (C460TR22270328, Cree Inc., USA), with dimensions of $220(W) \times 270$ $(L) \times 50(H) \mu \mathrm{m}^{3}$ and $460 \mathrm{~nm}$ in peak wavelength, are arranged in the array with the light-emitting surfaces facing downwards, allowing light to propagate through the holes without obstacles. Figure 1c, d shows the schematic diagram and photo of the reverse side view, where the 16-channel recording microelectrodes $(200 \mu \mathrm{m}$ in diameter) are distributed one-to-one next to the microLEDs and modified with iridium oxide $\left(\mathrm{IrO}_{x}\right)$ for lower impedance and higher signal-to-noise ratio (SNR) during neural recording. Finally, a reference electrode is integrated in the middle of the microelectrode array.

All the anodes of the micro-LEDs are individually addressable, whereas their cathodes are connected together to reduce the number of interconnection leads. For optogenetics, the activation of the introduced lightsensitive ion channels and pumps, channelrhodopsin-2, requires an optical stimulus wavelength of $473 \mathrm{~nm}^{25}$, which is in the wavelength range of the micro-LED. The assembled device can be attached to the unilateral cerebral cortex of a rat as shown in Fig. 1e. The depth in which micro-LEDs evoke neural activity across different layers is expected to be over $600 \mu \mathrm{m}$ as reported in the literature ${ }^{5}$. This design concept can be further expanded to larger or more complex areas, such as the cortical surface of nonhuman primates ${ }^{26}$ and the spinal cord and peripheral nervous system ${ }^{27}$, with more and smaller micro-LEDs and microelectrodes to realize more precise mapping, even utilizing color-tunable or multicolored micro-LEDs ${ }^{28,29}$ for simultaneous excitation and inhibition of neurons expressing different optogenetics opsins.

\section{Fabrication of the micro-LED array}

The micro-LED array fabrication process can be subdivided into 10 steps, as reported in our previous work ${ }^{30}$. As shown in Fig. 2a, a-1, a 500-nm-thick aluminum (Al) is evaporated on a silicon wafer by physical vapor deposition serving as a sacrificial layer ${ }^{31}$, then the bottom layer of a photosensitive polyimide (PI, Durimide 7505, Fujifilm, Japan) is spun at speeds of 1500 and $3000 \mathrm{rpm}$ on two separate wafers, followed by lithography and curing in $\mathrm{N}_{2}$ at $300{ }^{\circ} \mathrm{C}$ for $1 \mathrm{~h}$ to form approximately 5 and $2.5 \mu \mathrm{m}$ in 
thickness, respectively. $\mathrm{Cr} / \mathrm{Au}(20 / 300 \mathrm{~nm})$ layers are deposited, covered by a patterned positive photoresist ( 3 $\mu \mathrm{m}$ ), and etched using the ion-milling system (LKJ 150, Advanced Ion Beam Ltd, China) (Fig. 2a, a-2). The second PI layer is spun, patterned, and cured in $\mathrm{N}_{2}$ at $350{ }^{\circ} \mathrm{C}$ for 1 $\mathrm{h}$ to acquire another 5 or $2.5 \mu \mathrm{m}$ thickness (Fig. 2a, a-3). Then, a $50 \mu \mathrm{m}$-thick SU-8 2025 (MicroChem Corp., USA) is spun on top, photoetched, and developed to form a fence structure with the inner dimension of $240 \times 290$ $\mu \mathrm{m}^{2}$ and width of $50 \mu \mathrm{m}$, just above the aligned rectangular holes on the PI layers (Fig. 2a, a-4). The adhesion force between the SU-8 and PI is sufficient for the following operations to proceed without the occurrence of detachment. This structure is used for more accurate and easier alignment and placement of the micro-LED chips, and the effect without and with the SU-8 fence is further discussed in Supplementary Figure S1. A moderate droplet of transparent ultraviolet (UV) curable adhesive is applied and gathered around the inwall of the SU-8 fence (Fig. 2a, a-5). The micro-LED chips are lifted and placed one by one into the SU-8 fence with the gold bonding pads facing upwards (Supplementary Figure S1) (Fig. 2a, a-6). The adhesive is cured under UV light to keep the micro-LED chips firmly attached to the SU-8 fence and the PI substrate. Wire-bonding is utilized connect the two gold bond pads $(80 \mu \mathrm{m}$ in diameter, as the first bonding joint) of the micro-LED and the corresponding gold pads
( $160 \mu \mathrm{m}$ in diameter, as the second bonding joint) exposed on the top PI layer via the gold wire $(25 \mu \mathrm{m}$ in diameter) (Fig. 2a, a-7). Due to the poor adhesion of the second bonding joints and the gold pads on the flexible PI substrate, the conductive silver paste (H20E, Epoxy Technology Inc, USA) is dotted onto the second bonding joint and gold pads (Fig. 2a, a-8). After curing the silver paste at $80^{\circ} \mathrm{C}$ for $2 \mathrm{~h}$, the device is taken out and a moderate amount of transparent epoxy (PKM12C-1, Pattex, Germany) is applied to encapsulate the SU-8 fence, micro-LED and gold wires (Fig. 2a, a-9). Finally, the entire device is released from the wafer in the hydrochloric acid solution (Fig. 2a, a-10).

\section{Fabrication of the microelectrode array}

The fabrication process for the microelectrode array shares the first three steps similar to the micro-LED array, as shown in Fig. 2, b-1 to b-3. Then, a layer of positive photoresist $(30 \mu \mathrm{m})$ is spun and patterned to expose the $4 \times 4$ microelectrode sites (Fig. 2b, b-4). Ti/Ir (50/300 nm) layers are sputtered and patterned (Fig. 2b, b-5 and b-6) by lift-off in acetone to leave the microelectrode sites covered only by Ir. The array is released from the wafer in the hydrochloric acid solution and activated into iridium oxide, with a total thickness of 10 or $5 \mu \mathrm{m}$ (Fig. 2b, b-7). There are three typical $\mathrm{IrO}_{x}$ fabrication methods: sputtering iridium oxide film ${ }^{21}$; activated iridium oxide film ${ }^{32}$;
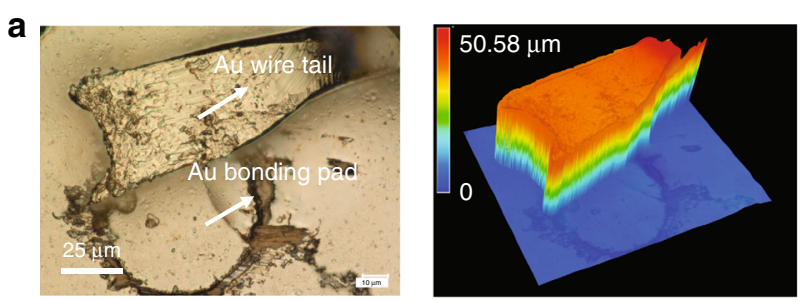

b
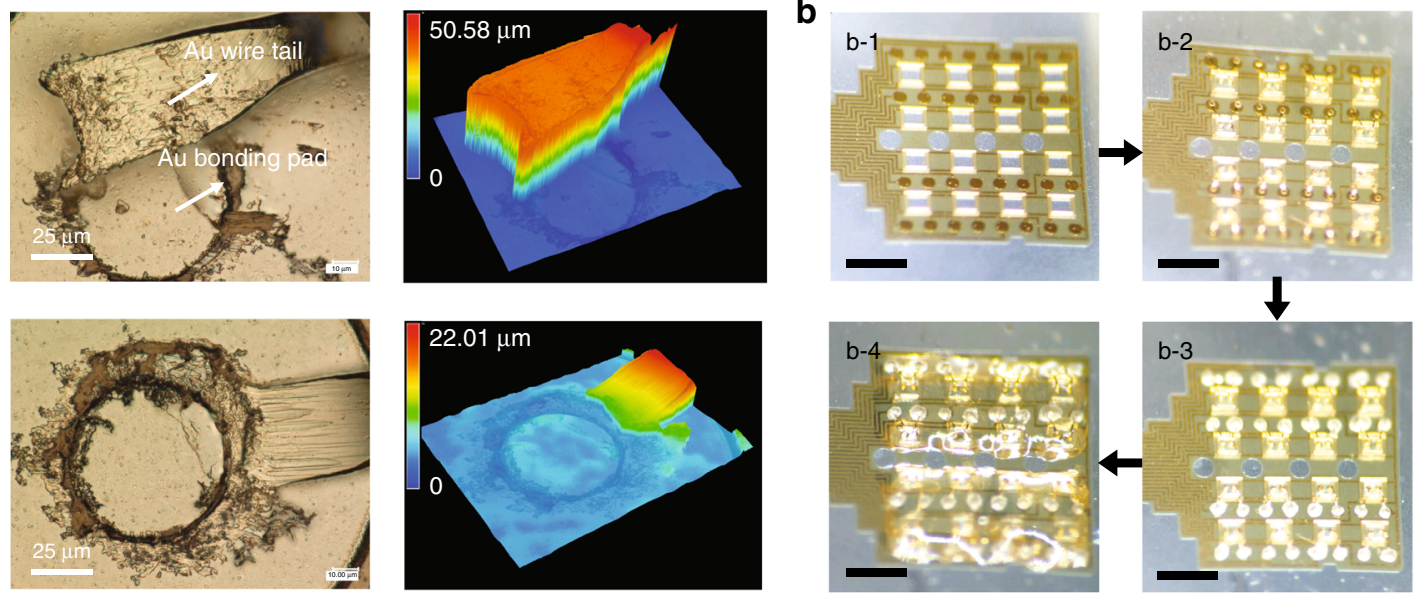

C
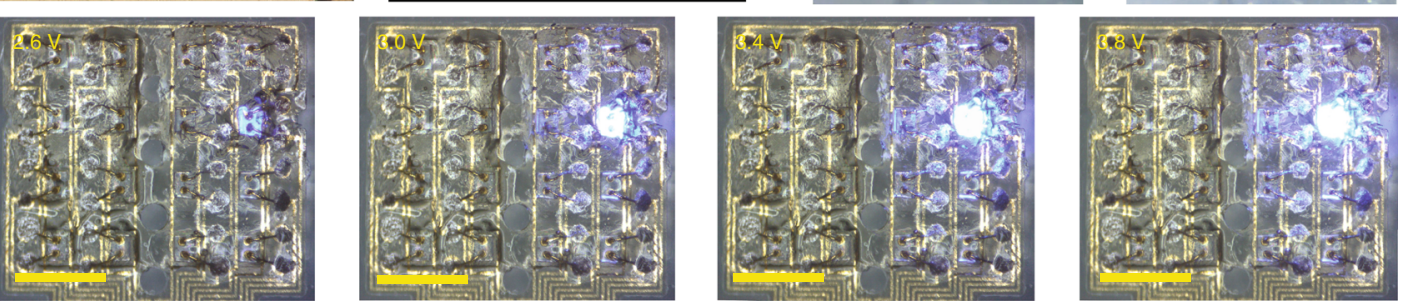

Fig. 3 Silver paste-aided wire-bonding on the flexible substrate and its effect. a Two states of second bonding joint on polyimide substrate: disconnected (above) and connected (below). b Flow photos of the micro-LEDs' setup and package showing the silver paste-aided wire-bonding. c Actual working effect of a single micro-LED under different voltages (2.6-3.8 V). All scale bars in $\mathbf{b}$ and $\mathbf{c}$ are $1 \mathrm{~mm}$ 

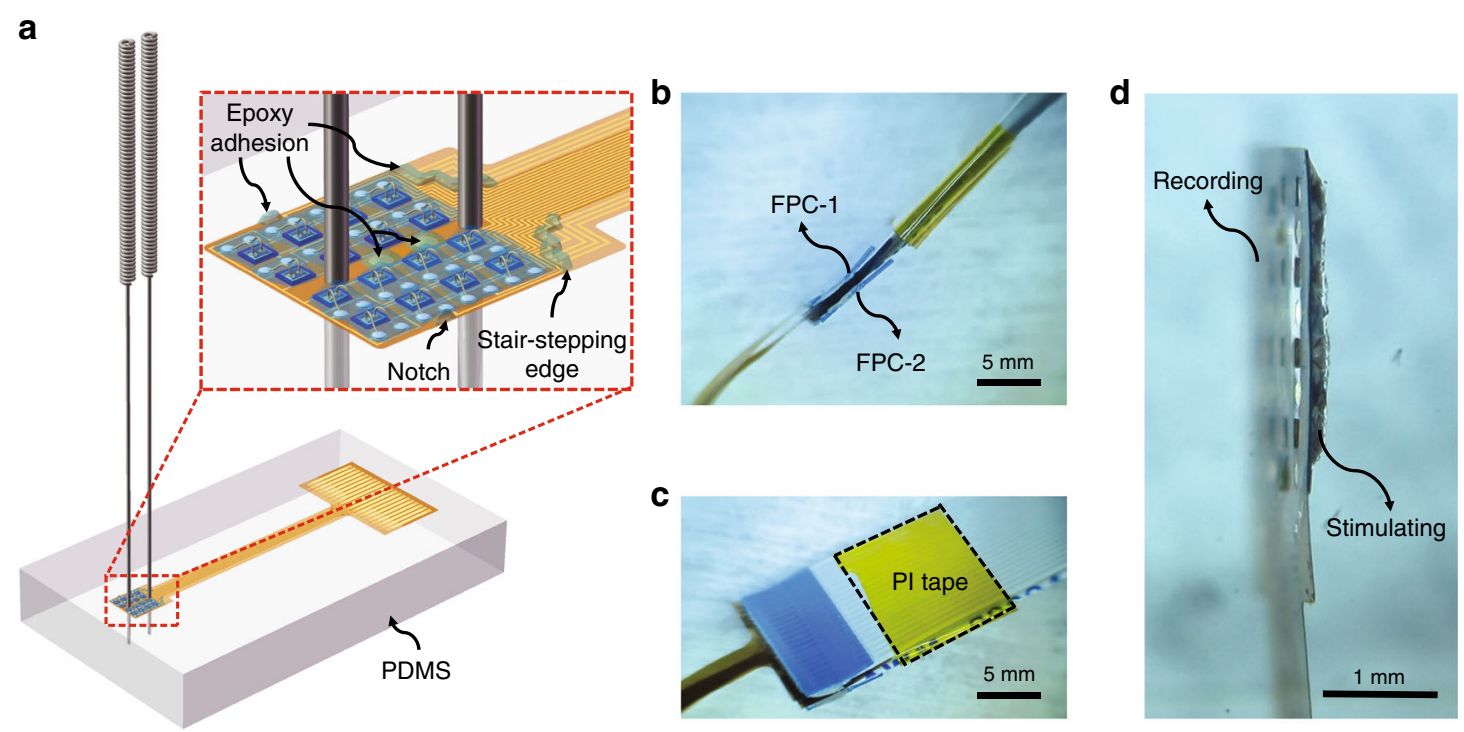

Fig. 4 System assembly. a Schematic diagram of back-to-back alignment of two sub-arrays with two needles inserted through the identical round holes into a PDMS layer. The inset shows the two sub-arrays assembled together using epoxy dispensed at reserved positions. $\mathbf{b}$ Two flexible printed circuit boards (FPC-1 and FPC-2) hot-pressed to the contact pads of the micro-LED array and microelectrode array, respectively. $\mathbf{c}$ Bond of two FPCs close to the interfaces with a PI tape. $\mathbf{d}$ Side view of the assembled device with hybrid recording and stimulating capabilities

and electrodeposited iridium oxide film ${ }^{33}$. Considering the necessity of the most stable and lowest phase response in the neural recording, the activated iridium oxide is the best choice with a sweeping potential from -1.0 to $+1.0 \mathrm{~V}$ at $0.05 \mathrm{~Hz}$ in PBS. After 300 activation cycles, the iridium is activated to iridium oxide. Finally, the two sub-arrays are glued together back to back by epoxy with the light of the micro-LEDs passing through the aligned openings, as illustrated in Fig. 2c.

\section{Silver paste-aided wire-bonding}

According to Hall et al. ${ }^{34}$, soft polymers absorb ultrasonic energy and deform under the compressive loading of a rigid capillary, thereby reducing the wire-bonding strength and yield. In practice, it is highly difficult to detach the tail from the stitch on a PI substrate, and the tail easily separates from the gold bonding pad even with relatively proper bonding parameters (ultrasonic power, pressure, time, and temperature). Two states of a second bonding joint are shown using a digital microscope (VHX-5000, KEYENCE, Japan) in Fig. 3a, disconnected (above) and connected (below) with the same bonding parameters. It was expected that a few wires would be bonded, but it was unreliable due to the weak bonding force. Thus, the weak interface between the tail of the gold wire and the exposed gold film area is the main reason for failure during wire-bonding, and no exfoliation of $\mathrm{Cr}$ adhesion layer from the PI substrate is observed. To address this issue, the conductive silver paste is dispensed on the gold pads on the PI. The improved wire-bonding process is shown in Fig. 3b, including fabrication of the SU-8 fence array above the aligned rectangular holes (b1), fixation of the micro-LED chips with UV curable adhesive in the SU-8 fences followed by wire-bonding (b2 ), reinforcement by the conductive silver paste (b-3), and encapsulation by epoxy (b-4). As clearly seen in Supplementary Figure S2, although the bonding wires (approximately $270 \mu \mathrm{m}$ in height) and epoxy encapsulation increase the total thickness (approximately $350 \mu \mathrm{m}$ in height) from the top, they do not interfere with the recording microelectrodes on the reverse side when in use. After release, the silver paste-aided wire-bonding performs well for all micro-LEDs, which are tested one by one with a final yield of $95 \%$. Three micro-LEDs failed on four samples in a total of 64 micro-LEDs due to the rupture of the exposed gold film or the connection of the micro-LED's cathode and anode when the silver paste was inattentively dispensed. Here the actual working effect of a single micro-LED is presented in Fig. 3c under different voltages $(2.6,3.0,3.4$, and $3.8 \mathrm{~V})$. The applied voltage upper limit is referred to the specifications of the microLED chip and the line resistance of the connection to the single micro-LED is approximately $40 \Omega$.

\section{Back-to-back alignment in assembly}

To easily assemble the micro-LED array and microelectrode array together, a back-to-back manual assembly method is proposed, which is less dependent on costly instruments such as a high-precision flip-chip bonder. Here an elastic, flat PDMS piece is utilized as a platform to 

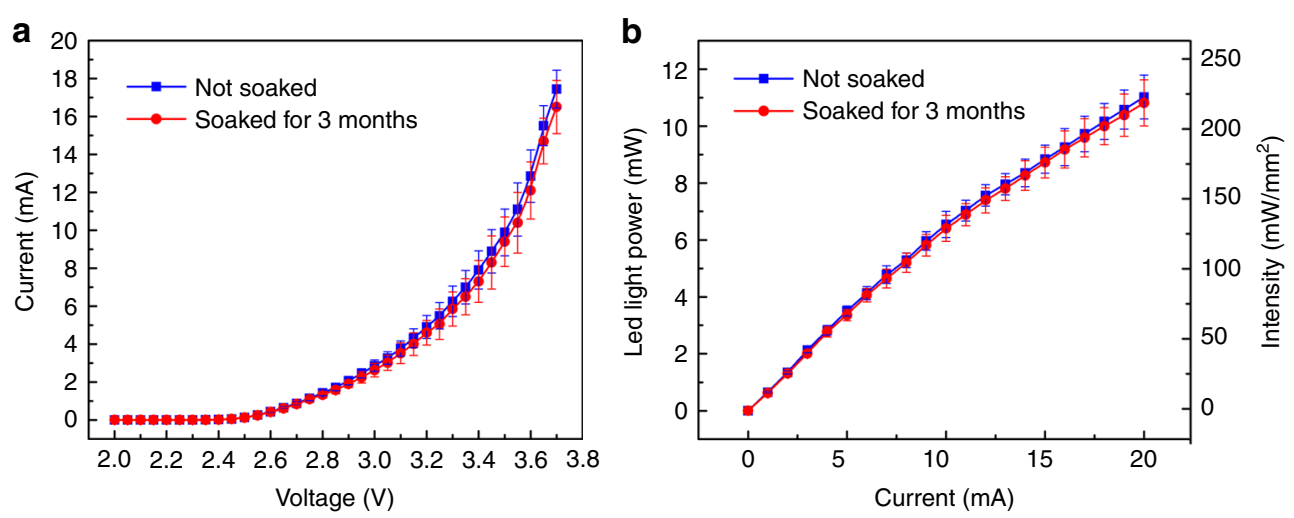

Fig. 5 Electrical and optical properties of micro-LED arrays soaked in PBS for $\mathbf{3}$ months. a $I-V$ mean value curves with standard deviations measured from 32 micro-LEDs on two samples. b Mean value and standard deviation of light output power and intensity of the same two samples

place and assist the assembly of the two sub-arrays. Figure $4 \mathrm{a}$ shows the schematic of the back-to-back alignment of the two sub-arrays with two steel needles inserted through the aligned round holes into the flat PDMS piece, in which the microelectrode array is beneath the microLED array with microelectrode sites facing the PDMS. The inset of Fig. 4a shows an enlarged view to show the epoxy distribution for adhesion, including the stair-stepping end edge, two identical round holes $(300 \mu \mathrm{m}$ in diameter) between the two needles, and two symmetrical notches at the edge on the top of the micro-LED array. These features are also shown in Figure S2c. The other ends of the two sub-arrays are separately hot-pressed to flexible printed circuits (FPC-1 and FPC-2) with the process described in Supplementary Figure S3. Then, the two FPCs are parallelly bonded together with the help of a thin PI tape (Fig. 4b, c). The side view of the final assembled device in Fig. $4 \mathrm{~d}$ clearly shows both the recording and stimulating sides.

\section{Results and discussion}

To verify the performance of the device, we studied the electrical, optical, and thermal properties of the microLED array, as well as the electrochemical, flexible, and mechanical properties of the microelectrode array. The results prove that this hybrid flexible neural system performs well and is promising for further animal experiments.

\section{Electrical and optical properties in a soaking test}

The electrical and optical properties of the wire-bonded micro-LEDs should be quantified for better use of the device with proper stimulation parameters. In addition, the uniformity and long-term stability of the micro-LEDs on the array should be evaluated. Here we picked two samples with 32 micro-LEDs in total and tested every single micro-LED before and after soaking in PBS for
3 months at room temperature under wet conditions, during which all micro-LEDs were only soaked without operation. The $I-V$ mean value curves with standard deviations were measured from the 32 micro-LEDs on the two samples by a semiconductor parameter analyzer (BA1500, Agilent Technologies, USA) as shown in Fig. 5a. The ratio of the standard deviation to mean value is $<13 \%$ and $17 \%$ before and after soaking, respectively, and the current slightly decreases after soaking. This may be due to the slight resistance increase $(<20 \Omega)$ in the electrode leads and the silver paste. For the optical measurement, the light output was evaluated using a spectrometer (QE65 Pro, Ocean Optics) and an integrating sphere (FOIS-1, Ocean Optics), and the intensity was calculated by dividing the effective emission area of the micro-LED $\left(190 \times 240 \mu \mathrm{m}^{2}\right)$. As shown in Fig. 5b, the mean values and standard deviations of the overlapping light power and intensity curves are obtained from these two samples with the 32 micro-LEDs. Similarly, the ratio of the standard deviation to mean value is $<7 \%$ and $8 \%$ before and after soaking, respectively, and the intensity shows a slight decrease after soaking as well. Considering the minimum light energy density of $1 \mathrm{~mW} / \mathrm{mm}^{2}$ to induce neuronal action potentials ${ }^{1}$, the light intensity provided by our micro-LED could be sufficient for neurons in deeper layers. Our results indicate that the micro-LED array is uniform and reliable for long-term use in a liquid environment.

\section{Thermal property of the integrated micro-LED}

Brain tissue damage caused by the heat production of the micro-LED is a major concern for in vivo applications. Accordingly, the temperature variation of a single microLED on the array is investigated using a thermal infrared (IR) imager (Fluke TiX 560, USA) with $0.05 \mathrm{~K}$ resolution and macro IR lens (Fluke Lens/25 Mac2, USA) with a pixel size as small as 25 microns. The test was performed 

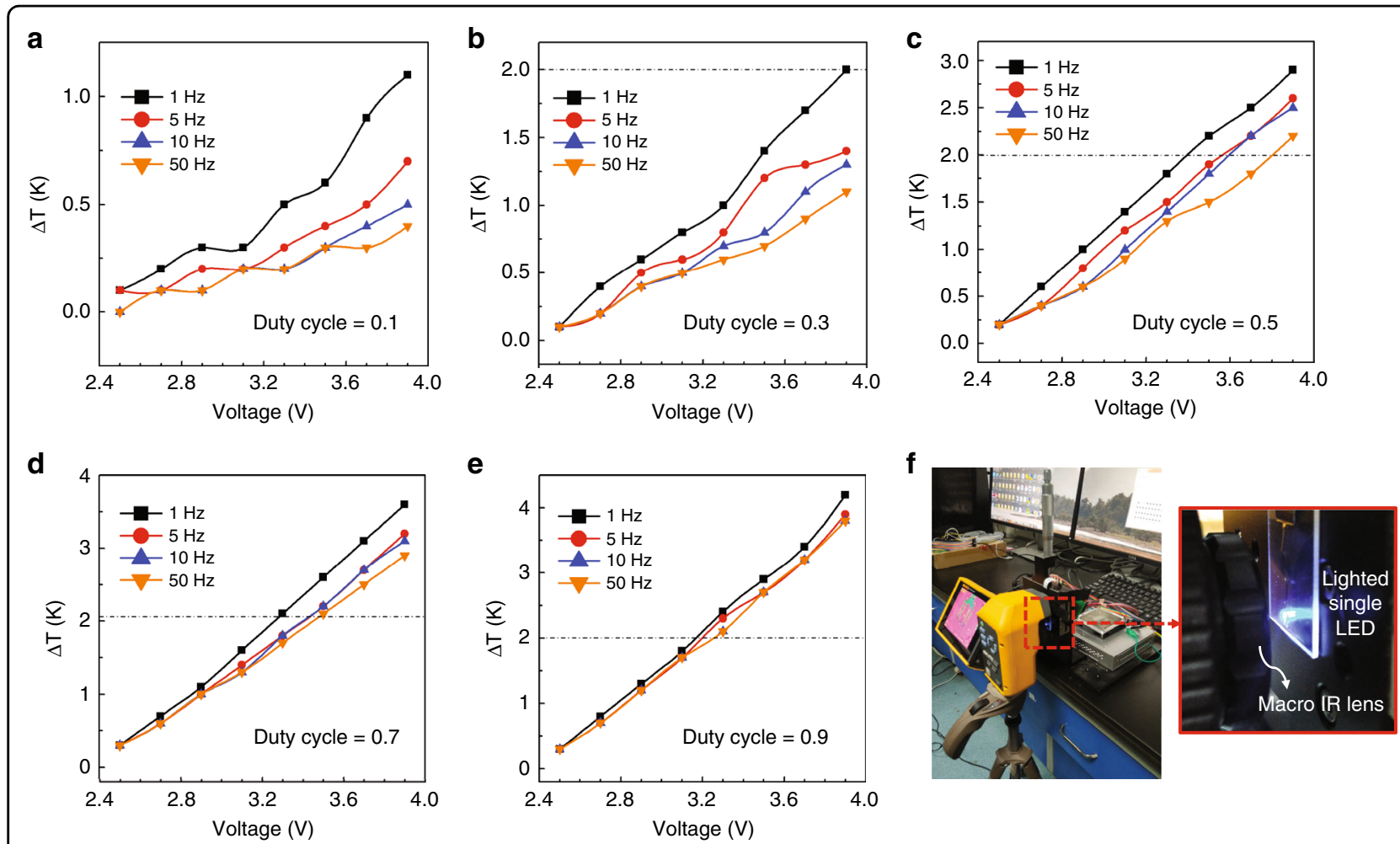

Fig. 6 Thermal property of the integrated single micro-LED on the array. Duty cycle from 10-90\% (a-e), frequency from 1 to $50 \mathrm{~Hz}$, and voltage from 2.5 to $3.9 \mathrm{~V}$; dashed line at $\Delta \mathrm{T}=2 \mathrm{~K}$ indicating the maximum acceptable temperature increase to avoid thermal damage of brain tissue. f Measurement setup with an IR thermal imager with the enlarged view of the micro-LED array facing the macro IR lens

in an enclosed environment with a constant room temperature at $20^{\circ} \mathrm{C}$ where ventilation was strictly controlled. With the voltage increasing from 2.5 to $3.9 \mathrm{~V}$ and different frequencies ranging from 1 to $50 \mathrm{~Hz}$, the rise in temperature of the single micro-LED was measured on the array floating in air with duty cycles from 0.1 to 0.9 , corresponding to Fig. 6a-e. The dashed line indicates the maximal acceptable temperature increase $(\Delta T=2 \mathrm{~K})$ for brain implants ${ }^{8}$, which serves as a meaningful reference when conducting animal experiments. As the applied voltage increases, the temperature simultaneously increases. The difference in the temperature increase at varied frequencies is not obvious. However, the temperature increase distinctly rises with the duty cycle. The increase of duty cycle leads to an increased accumulation of heat in the micro-LED chip with less time to dissipate it. Therefore, the influence of the duty cycle is larger than that of the frequency. Figure $6 \mathrm{f}$ exhibits the measurement setup with an IR thermal imager, a micro-LED array fixed on a three-axis platform, and an LED power supply. The luminous surfaces of the micro-LEDs face the macro IR lens as shown in the enlarged view of Fig. $6 \mathrm{f}$. One thing to note here is that the luminous surface of the micro-LED is not in direct contact with the brain tissue due to the clearance resulting from the thickness of the subjacent microelectrode array. Therefore, the temperature increase at the cortical surface should be lower, which implies that it is safe to apply a voltage higher than the maximal allowed value shown here.

\section{Electrochemical property of microelectrodes and stability}

The microelectrode sites are modified with activated $\mathrm{IrO}_{x}^{32}$ to improve the electrochemical performances at the electrode-tissue interface. Low microelectrode impedance is beneficial for acquiring high SNR in neural recording. Due to the likely friction or pressure-lead exfoliation of $\mathrm{IrO}_{x}$ during attachment and long-term use in animals, its stability is verified by comparing the cyclic voltammetry (CV), impedance, and phase in electrochemical measurements before and after 5000 iterations of friction or pressing (Fig. $7 \mathrm{a}-\mathrm{c}$ ). To elucidate the necessity of modification, bare gold microelectrodes $(200 \mu \mathrm{m}$ in diameter $)$ are also tested as a comparison. Here four $\mathrm{IrO}_{x}$-modified microelectrode arrays and two bare gold arrays are chosen for the test. The $\mathrm{IrO}_{x}$-modified microelectrode arrays were measured before any operation, in which two of them underwent friction back and forth ${ }^{21}$, and the other two were under repeated compression ${ }^{30}$, both on a flat agar gel for 5000 iterations. The friction provides shear stress that is applied parallel to the surface of the microelectrode sites 

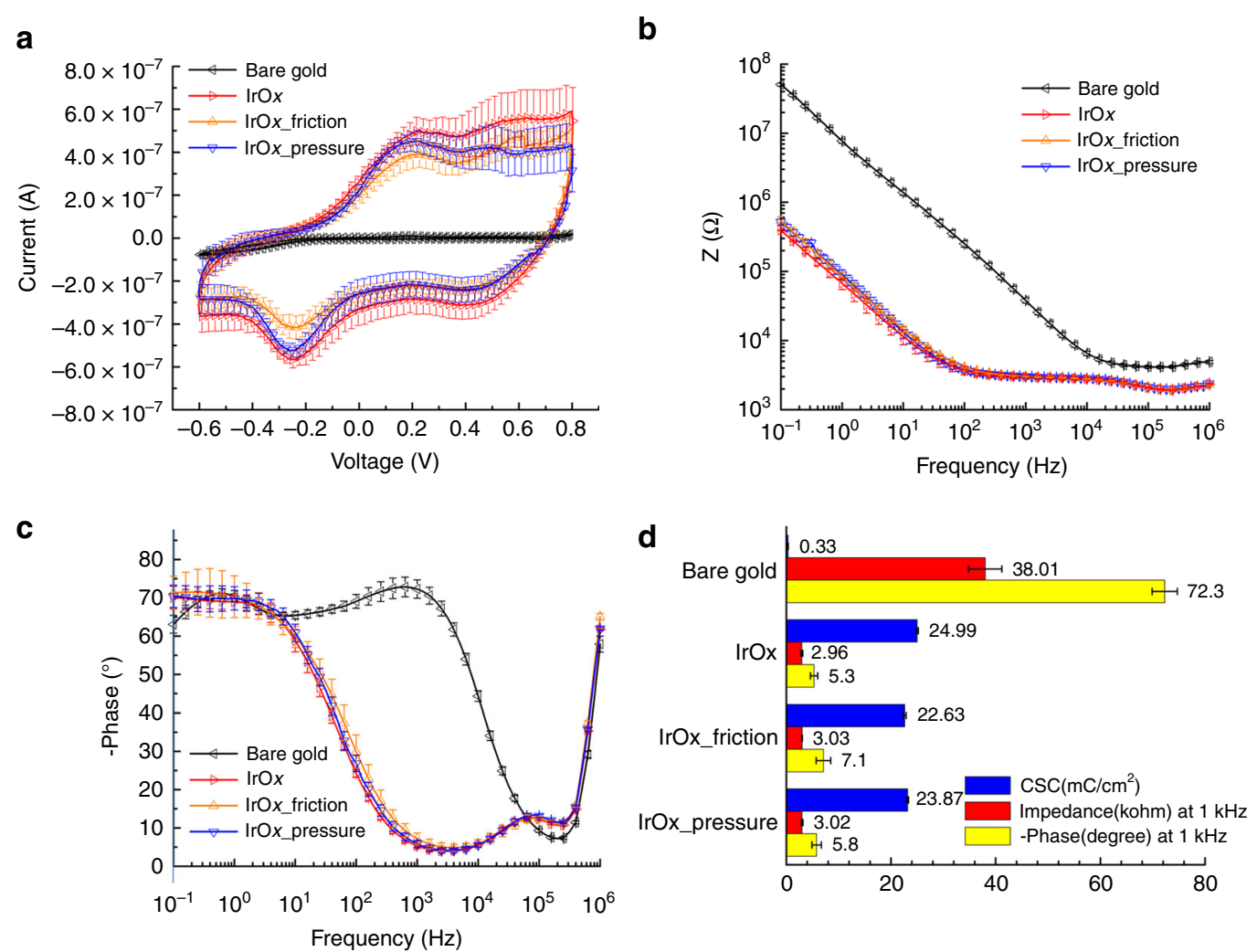

Fig. 7 Electrochemical properties of IrO $_{\mathbf{x}}$-modified microelectrodes and their stability. $\mathbf{a}$ Cyclic voltammogram, $\mathbf{b}$ impedance, and $\mathbf{c}$ phase of two $\mathrm{IrO}_{x}$-modified microelectrode arrays (32 sites) before and after 5000 iterations of friction or pressing, compared with bare gold microelectrodes. d Comparison of bare gold and $\mathrm{IrO}_{x}$-modified microelectrodes before and after friction or pressing in charge storage capacity (CSC) as well as impedance and phase at $1 \mathrm{kHz}$

with no additional pressure from the top, which imitates the actual relative movement to the brain tissue. In addition, the nonnegligible swelling of brain tissue underneath the recording sites is also simulated by repeated lifting and pressing of a cotton swab on the microelectrode array with an average pressure of approximately $180 \mathrm{kPa}$. After, the arrays were measured again in PBS using a standard three electrodes system (PGSTAT12 Autolab, EcoChemie, Utrecht, Netherlands) with a saturated calomel electrode as the reference and a Pt sheet as the counter electrode. The mean value curves with standard deviations not only illustrate the uniformity of the 32 tested microelectrode sites and stability of $\operatorname{IrO}_{x}$ but also prove the great improvement in the electrochemical properties as a result of $\mathrm{IrO}_{x}$.

The charge storage capacity (CSC) is calculated from the $\mathrm{CV}$ in Fig. $7 \mathrm{a}$, and values for the electrodes with bare gold, $\mathrm{IrO}_{x}, \mathrm{IrO}_{x}$ after friction, and $\mathrm{IrO}_{x}$ after pressing are compared in Fig. $7 \mathrm{~d}$ and are $0.33 \pm 0.05,24.99 \pm 0.22$, $22.63 \pm 0.27$, and $23.17 \pm 0.19 \mathrm{mC} / \mathrm{cm}^{2}$, respectively, in correspondence with previous results ${ }^{32,35}$. The CSC of the $\mathrm{IrO}_{x}$-coated electrode is approximately 76 times higher than that of the bare gold electrode and there are only a
10.4 and $7.3 \%$ decrease after friction and pressing. The impedance and phase at $1 \mathrm{kHz}$ are presented in Fig. $7 \mathrm{~d}$. The impedance values are $38.01 \pm 3.18,2.96 \pm 0.21,3.03 \pm$ 0.06 , and $3.02 \pm 0.16 \mathrm{k} \Omega$ and the phases are $-72.3 \pm 2.4^{\circ}$, $-5.3 \pm 0.7^{\circ},-7.1 \pm 1.4^{\circ}$, and $-5.8 \pm 0.9^{\circ}$ for the electrodes with bare gold, $\mathrm{IrO}_{x}, \mathrm{IrO}_{x}$ after friction, and $\mathrm{IrO}_{x}$ after pressure, respectively. These values are comparable to previous studies ${ }^{32,36}$. The impedance and phase of the $\mathrm{IrO}_{x}$-coated electrodes are less than one-tenth of bare gold, which are lowered by the cyclic activation and oxidation of Ir in PBS. In addition, no obvious change in the impedance or the phase is observed after friction and pressing.

\section{Flexibility of the microelectrode array}

To study the flexibility of the two PI microelectrode arrays with thicknesses of 5 and $10 \mu \mathrm{m}$, the attachment experiment was conducted on cylindrical Teflon tubes with diameters of 1.5, 2, 3, and $4 \mathrm{~mm}$ as shown in Fig. 8a. These simplified cylinder models are small enough to be meaningful references to the cortex surface of a rat and easier to quantitatively study the flexibility. The red and green dots represent unwrapped and wrapped states, 
a

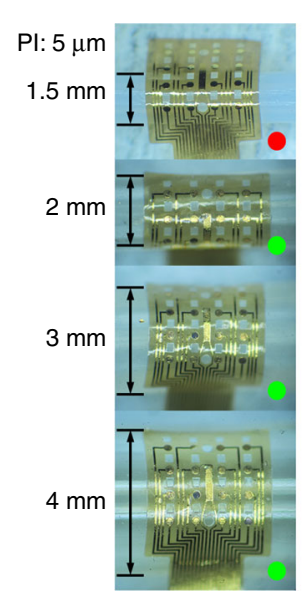

PI: $10 \mu \mathrm{m}$

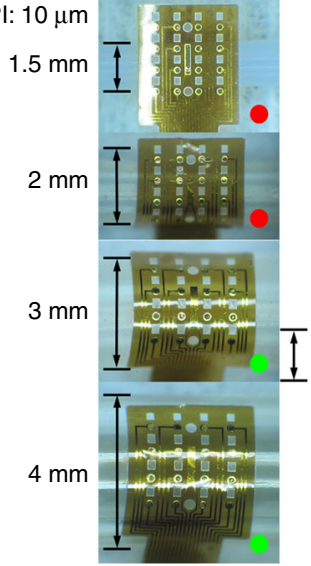

c

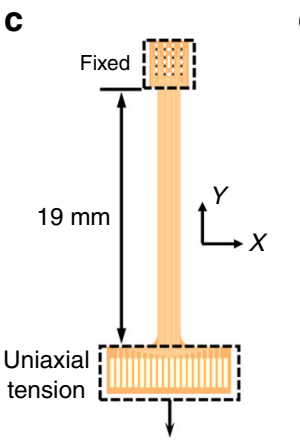

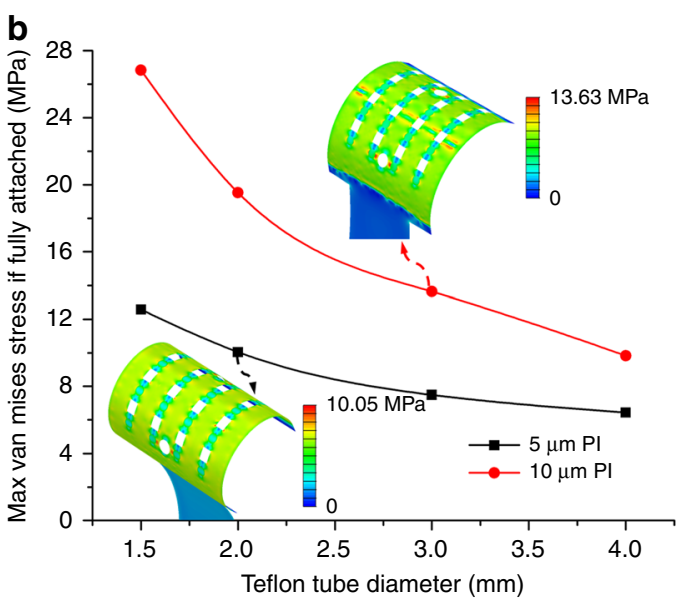

e

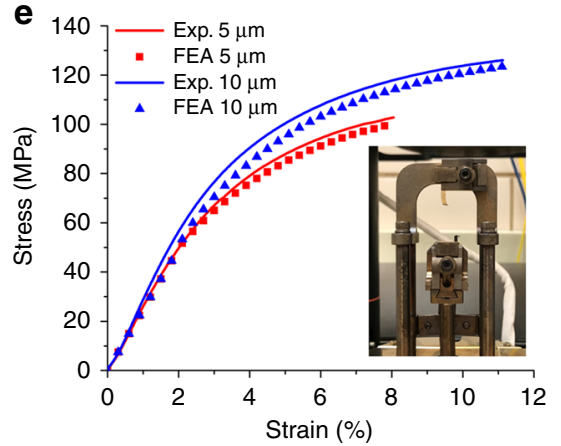

Fig. 8 Flexibility and mechanical property of the microelectrode arrays with an overall thickness of $\mathbf{5}$ or $\mathbf{1 0} \boldsymbol{\mu m}$. a Attachment experiments of PI microelectrode arrays on Teflon tubes with different diameters of 1.5, 2, 3, and $4 \mathrm{~mm}$. The red and green dots represent poor and good contact, respectively. $\mathbf{b}$ The simulated maximal stresses of the arrays with different thicknesses when fully attached to the Teflon tubes with the same diameters. The insets show the stress distribution of the fully attached $5 \mu \mathrm{m} \mathrm{PI}$ on a $2 \mathrm{~mm}$ tube and $10 \mu \mathrm{m} \mathrm{PI}$ on a $3 \mathrm{~mm}$ tube, respectively. c The diagram of the uniaxial tensile test of the microelectrode array with one side fixed and the other side under uniaxial tension. $\mathbf{d}$ Stress distribution of the PI microelectrode array with a thickness of $10 \mu \mathrm{m}$ at the fracture critical state. e Experimental and FEA results of strain vs. stress curves of 5- and $10-\mu m$-thick arrays. The inset shows the broken specimen in the DMA

respectively. With only water capillarity as the adhesion force $^{37}$, the thinner $5 \mu \mathrm{m}$ PI achieves excellent conformal contact when the cylinder diameter is not $<2 \mathrm{~mm}$, whereas the thicker $10 \mu \mathrm{m}$ PI can only wrap well on cylinders with diameters $\geq 3 \mathrm{~mm}$.

To reveal the adhesion forces applied to the tubes, it was assumed that these two arrays could be fully attached on Teflon tubes and the maximum van Mises stresses were calculated in the finite element analysis (FEA) software ABAQUS (SIMULIA, Pawtucket, RI, USA) (Fig. 8b). The film devices were modeled by four-node composite shell elements (S4R) and the tubes were modeled by the hexahedron three-dimensional solid element (C3D8R). The surface traction was applied on the array area to realize complete contact with the tube. A displacement boundary restriction was applied to the middle line of the rectangle array area in the $x$-direction to keep the array facing the tube without rotation or misalignment. The insets of Fig. $8 \mathrm{~b}$ show the van Mises stress distribution of the $5 \mu \mathrm{m}$
PI when fully attached to a $2 \mathrm{~mm}$ tube and the $10 \mu \mathrm{m}$ PI to a $3 \mathrm{~mm}$ tube corresponding to the minimum conformal wrapping curvatures observed in experiment. The maximum stresses are 10.05 and $13.63 \mathrm{MPa}$, respectively.

\section{Mechanical property of the microelectrode array}

When conducting the animal experiment, the manual operation or stiffness mismatch between the flexible device and covered dental cement may induce a drawing force on the electrode. Therefore, it is necessary to evaluate the mechanical property to avoid tensile failure. Here two PI microelectrode arrays with thicknesses of 5 and $10 \mu \mathrm{m}$ were tested. The sample on one side was fixed, whereas the other side was movable between the clamps in the dynamic thermomechanical analysis system (DMA Q800, TA Instrument, USA). The load was uniaxially applied from both sides at an initial length of $19 \mathrm{~mm}$ as shown in Fig. 8c. The ABAQUS enables studies of the mechanical response of the film devices under tension. They were modeled by 
shell elements with the entire array fixed in the upper dotted box and the uniaxial downward tensile force from the upper edge of the connection pad in the lower dotted box as shown in Fig. 8c. The 10- $\mu$ m-thick specimen with the van Mises stress distribution when stretched to the critical fracture state is illustrated in Fig. $8 \mathrm{~d}$. The fracture first occurs on either end of the stripe due to the stress concentration in the connecting regions of the stripe and two ends, as shown in the enlarged views of Fig. 8d.

The experimental and FEA results of strain vs. stress are compared in Fig. 8e for both the 5 and $10 \mu \mathrm{m}$ films. The experimental strain-stress curves show almost overlapping elastic deformation segments for the two specimens. Young's modulus of $E=2.5 \mathrm{GPa}$ can be calculated based on the experimental data. In the experiments, the crosssectional area rapidly experienced necking in a localized region of the specimen when the loading was continued beyond the ultimate stress. The strains reached $8.0 \%$ and $11.1 \%$ for the 5 and $10 \mu \mathrm{m}$ specimens, respectively, and the stresses reached 102.8 and $126 \mathrm{MPa}$ when the fracture occurred, respectively. The thicker PI film has a larger elastic range than that of the thinner film. Additionally, the characteristics include elastic and plastic behaviors, but it is observably without the yield strength point due to the material properties. The FEA results match well with the experimental data from the elastic to necking state.

The tradeoff between mechanical strength and flexibility should be taken into consideration. As reported in a previous study ${ }^{30}$, the thinner sub-arrays can be independently attached on the cortical surface one by one without assembly in acute animal experiments, with all microLEDs precisely aligned to ensure that the light directly passes through the holes. However, to ensure adequate mechanical strength and avoid tensile failure in chronic implantation for freely moving animals, the thicker assembled sub-array is a better choice.

\section{Conclusions}

In this paper, the design, fabrication, and characterization of an opto-electric interface combining $4 \times 4$ wirebonded micro-LEDs and $4 \times 4 \mathrm{IrO}_{x}$-modified microelectrodes for simultaneous light stimulation and neural recording are presented. A key advantage of this novel design is that it enables the light of the micro-LED to directly pass through the holes without loss while using the microelectrode array to realize local mapping. With the use of the silver paste-aided wire-bonding method, the micro-LED array functions well. The uniformity of all micro-LEDs on the array and long-term stability after soaking also perform well. In addition, the thermal properties of the micro-LED on the array in this study is useful as a reference for light stimulation to avoid thermal damage to local tissue. The stability of activated $\mathrm{IrO}_{x^{-}}$ modified microelectrodes is validated by 5000 iterations of pressing or friction and the electrochemical performance declines very little. The 5 - $\mu \mathrm{m}$-thick microelectrode array achieves excellent conformal contact when the cylinder diameter is not $<2 \mathrm{~mm}$, whereas the $10-\mu \mathrm{m}$-thick array can only wrap well on cylinders with diameters $\geq 3 \mathrm{~mm}$. For the mechanical strength of the 5- and 10- $\mu \mathrm{m}$-thick devices, the stress reaches 102.8 and $126 \mathrm{MPa}$ when fracture occurs, respectively. Owing to the widespread study of optogenetics in neural science and diseases, we believe this study will provide a useful, functionally integrated tool and insight to produce more precise optoelectric neural interfaces.

\begin{abstract}
Acknowledgements
This work was partially funded by the National Key R\&D Program of China under grant 2017YFB1002501, the National Natural Science Foundation of China (No. 51475307 and 61728402), the Research Program of Shanghai Science and Technology Committee (17JC1402800 and 15JC1400103), the Program of Shanghai Academic/Technology Research Leader (18XD1401900), ZBYY-MOE Joint Funding (6141 A02022604), and the China Scholarship Council (201606230100). The authors thank the Shanghai Institutes for Biological Sciences, Chinese Academy of Sciences. The authors are also grateful to the Center for Advanced Electronic Materials and Devices (AEMD) of Shanghai Jiao Tong University.
\end{abstract}

\section{Author details \\ 'National Key Laboratory of Science and Technology on Micro/Nano Fabrication, Department of Micro/Nano Electronics, Shanghai Jiao Tong University, Shanghai 200240, China. ${ }^{2}$ Electrical and Computer Engineering Department, Michigan State University, East Lansing, MI 48824, USA}

\section{Conflict of interest}

The authors declare that they have no conflict of interest.

Supplementary information accompanies this paper at https://doi.org/ 10.1038/s41378-018-0027-0.

Received: 1 March 2018 Revised: 6 June 2018 Accepted: 6 July 2018 Published online: 08 October 2018

\footnotetext{
References

1. Boyden, E. S. et al. Millisecond-timescale, genetically targeted optical control of neural activity. Nat. Neurosci. 8, 1263-1268 (2005).

2. Adamantidis, A. R. et al. Neural substrates of awakening probed with optogenetic control of hypocretin neurons. Nature 450, 420-424 (2007).

3. Sparta, D. R. et al. Construction of implantable optical fibers for long-term optogenetic manipulation of neural circuits. Nat. Protoc. 7, 12-23 (2012).

4. Clements, I. P. et al. Miniaturized LED sources for in vivo optogenetic experimentation. Optogenetics 8586, 85860X (2013).

5. Kwon, K. Y. et al. Opto- $\mu E C o G$ array: a hybrid neural interface with transparent $\mu E C O G$ electrode array and integrated LEDs for optogenetics. IEEE Trans. Biomed. Circuits Syst. 7, 593-600 (2013).

6. Zorzos, A. N. et al. Three-dimensional multiwaveguide probe array for light delivery to distributed brain circuits. Opt. Lett. 37, 4841-4843 (2012).

7. Lee, J. et al. Transparent intracortical microprobe array for simultaneous spatiotemporal optical stimulation and multichannel electrical recording. Nat. Methods 12, 281-313 (2015).

8. Kim, T. I. et al. Injectable, cellular-scale optoelectronics with applications for wireless optogenetics. Science 340, 211-216 (2013).

9. Park, S. I. et al. Ultraminiaturized photovoltaic and radio frequency powered optoelectronic systems for wireless optogenetics. J. Neural Eng. 12, 056002 (2015).

10. Leuthardt, E. C. et al. A brain-computer interface using electrocorticographic signals in humans. J. Neural Eng. 1, 63-71 (2004).

11. Schalk, G. \& Leuthardt, E. C. Brain-computer interfaces using electrocorticographic signals. IEEE Rev. Biomed. Eng. 4, 140-154 (2011).
} 
12. Richner, T. J. et al. Optogenetic micro-electrocorticography for modulating and localizing cerebral cortex activity. J. Neural Eng. 11, 016010 (2014).

13. Ledochowitsch, P. et al. Strategies for optical control and simultaneous electrical readout of extended cortical circuits. J. Neurosci. Methods 256, 220-231 (2015).

14. Fukushima, M., Chao, Z. C. \& Fujii, N. Studying brain functions with mesoscopic measurements: advances in electrocorticography for non-human primates. Curr. Opin. Neurobiol. 32, 124-131 (2015).

15. Park, A. H. et al. Optogenetic mapping of functional connectivity in freely moving mice via insertable wrapping electrode array beneath the skull. ACS Nano 10, 2791-2802 (2016).

16. Park, D. W. et al. Graphene-based carbon-layered electrode array technology for neural imaging and optogenetic applications. Nat. Commun. 5, 5258 (2014).

17. Yazdan-Shahmorad, A. et al. A large-scale interface for optogenetic stimulation and recording in nonhuman primates. Neuron 89, 927-939 (2016).

18. Yoshimoto, S. et al. Implantable wireless 64-channel system with flexible ECoG electrode and optogenetics probe. In IEEE Biomedical Circuits and System Conference 476-479 (IEEE, Shanghai, 2016).

19. Goßler, C. et al. GaN-based micro-LED arrays on flexible substrates for optical cochlear implants. J. Phys. D Appl. Phys. 47, 205401 (2014).

20. Xiang, Z. L., Liu, J. Q. \& Lee, C. K. A flexible three-dimensional electrode mesh: an enabling technology for wireless brain-computer interface prostheses. Microsyst. Nanoeng. 2, 16012 (2016).

21. Ji, B. W. et al. Photoelectric neural interface combining wire-bonding $\mu$ LEDs with iridium oxide microelectrodes for optogenetics. In IEEE International Conference on Micro Electro Mechanical Systems 538-541 (IEEE, Las Vegas, 2017).

22. Ayub, S. et al. An intracerebral probe with integrated $10 \times 1 \mu \mathrm{LED}$ array for optogenetic experiments at $460 \mathrm{~nm}$. Procedia Eng. 120, 472-475 (2015).

23. Slutzky, M. W. et al. Optimal spacing of surface electrode arrays for brain-machine interface applications. J. Neural Eng. 7, 026004 (2010).

24. Kwon, K. Y. et al. Opto- $\mu E C o G$ array: transparent $\mu E C o G$ electrode array and integrated LEDs for optogenetics. In IEEE Biomedical Circuits and System Conference 164-167 (IEEE, Hsinchu, 2012).
25. Zhang, F. et al. Multimodal fast optical interrogation of neural circuitry. Nature 446, 633-639 (2007).

26. Ruiz, O. et al. Optogenetics through windows on the brain in the nonhuman primate. J. Neurophysiol. 110, 1455-1467 (2013).

27. Park, S. I. et al. Soft, stretchable, fully implantable miniaturized optoelectronic systems for wireless optogenetics. Nat. Biotechnol. 33, 1280-1286 (2015).

28. Lee, $\mathrm{K}$. et al. Flexible inorganic light emitting diodes and transparent PEDOT: PSS/Parylene C for simultaneous optogenetics and electrocorticography. In Society of Photo-optical Instrumentation Engineers. International Society for Optics and Photonics (Conference Presentation) 1005208-1 (SPIE, San Diego, 2017). Editors: Samarendra K. Mohanty; Nitish V. Thakor; E. Duco Jansen.

29. Ayub, S. et al. Compact intracerebral probe with yellow phosphor-based light conversion for optogenetic control. In IEEE International Conference on Solid-State Sensors, Actuators and Microsystems 1703-1706 (IEEE, Kaohsiung, 2017).

30. Ji, B. W. et al. Flexible optoelectric neural interface integrated wire-bonding $\mu \mathrm{LEDs}$ and microelectrocorticography for optogenetics. IEEE Trans. Electron Devices 64, 2008-2015 (2017).

31. Lee, S. et al. Development of battery-free neural interface and modulated control of tibialis anterior muscle via common peroneal nerve based on triboelectric nanogenerators (TENGS). Nano Energy 33, 1-11 (2017).

32. Kang, $X$. Y. et al. Controlled activation of iridium film for AIROF microelectrodes. Sens. Actuators B Chem. 190, 601-611 (2016).

33. Lee, $S$. et al. Toward bioelectronic medicine-neuromodulation of small peripheral nerves using flexible neural clip. Adv. Sci. 4, 1700149 (2017).

34. Hall, E., Lyons, A. M. \& Weld, J. D. Gold wire bonding onto flexible polymeric substrates. IEEE Trans. Compon. Packag. Manuf. Technol. A 19, 12-17 (1996).

35. Shin, S. et al. High charge storage capacity electrodeposited iridium oxide film on liquid crystal polymer-based neural electrodes. Sens. Mater. 3, 243-260 (2016).

36. Ganji, M. et al. Scaling effects on the electrochemical performance of poly (3, 4-ethylenedioxythiophene (PEDOT), Au, and Pt for electrocorticography recording. Adv. Funct. Mater. 27, 1703018 (2017).

37. Kim, D. H. et al. Dissolvable films of silk fibroin for ultrathin, conformal biointegrated electronics. Nat. Mater. 9, 511-517 (2010). 